Document downloaded from:

http://hdl.handle.net/10251/78688

This paper must be cited as:

Tortajada-Genaro, LA.; Mena-Mollá, S.; Niñoles Rodenes, R.; Puigmule, M.; Viladevall, L.; Maquieira Catala, Á. (2016). Genotyping of single nucleotide polymorphisms related to attention-deficit hyperactivity disorder. Analytical and Bioanalytical Chemistry. 408(9):23392345. doi:10.1007/s00216-016-9332-3.

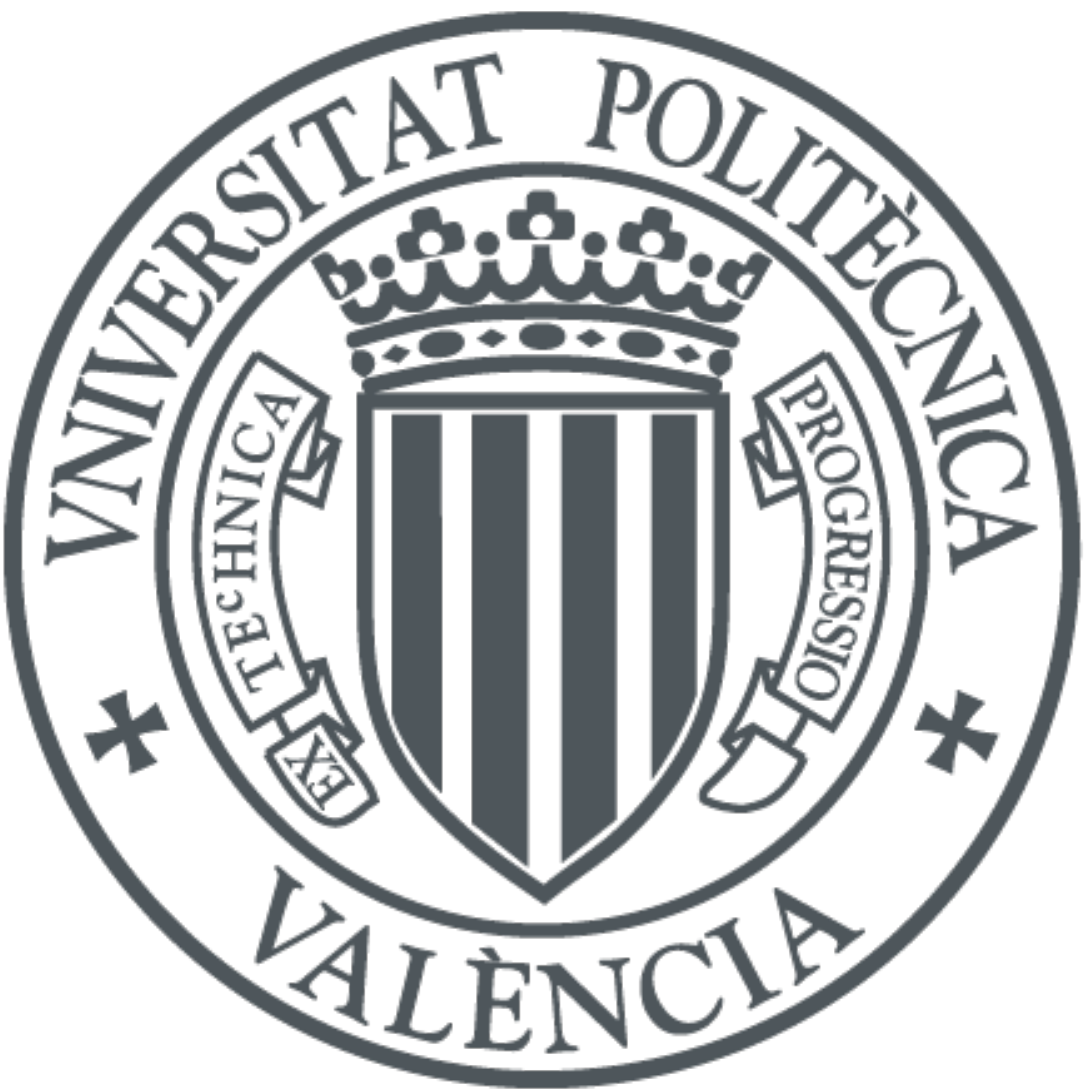

The final publication is available at

http://dx.doi.org/10.1007/s00216-016-9332-3

Copyright Springer Verlag (Germany)

Additional Information 


\section{Genotyping of single nucleotide polymorphisms related to attention deficit hyperactivity disorder}

Luis A. Tortajada-Genaro ${ }^{1}$, Salvador Mena ${ }^{1}$, Regina Niñoles ${ }^{1}$, Marta Puigmule ${ }^{2}$, Laia Viladevall $^{2}$, Ángel Maquieira ${ }^{1}$

5

(1) Centro de Reconocimiento Molecular y Desarrollo Tecnológico (IDM) - Departamento de Química, Universitat Politècnica de València, Camino de Vera s/n, E46022 Valencia, Spain

(2) AB-Biotics SA (Barcelona, Spain)

\section{ABSTRACT}

Pharmacological treatment of several diseases, such as attention deficit hyperactivity disorder (ADHD), presents marked variability in efficiency and its adverse effects. The genotyping of specific single nucleotide polymorphisms (SNPs) can support the prediction of responses to drugs and the genetic risk of presenting comorbidities associated with ADHD. This study presents two rapid and affordable microarray-based strategies to discriminate three clinically important SNPs in genes ADRA2A, SL6CA2, and OPRM1 (rs1800544, rs5569, and rs1799971, respectively). These approaches are allele-specific oligonucleotide hybridization (ASO), and a combination of allele-specific amplification (ASA) and solid-phase hybridization. Buccal swab and blood samples taken from ADHD patients and controls were analyzed by ASO, ASA and a gold-reference method. The results indicated that ASA is superior in genotyping capability and analytical performance.

Keywords: SNP genotyping, low-cost microarray technology, pharmacogenetics, attention deficit hyperactivity disorder. 


\section{Highlights}

Pharmacogenetic genotyping easy-going tool

Microarray assay for doctor office applications

30 Screening of SNP related to a psychiatric disorder on polymer chip 


\section{INTRODUCTION}

Attention-deficit/hyperactivity disorder (ADHD), a neurodevelopmental disorder, is characterized by inattention, hyperactivity and impulsive behavior. This disease is a highly prevalent childhood-onset neuropsychiatric condition (about 5\% in schoolchildren), and is also detected in adults $[1,2]$. One major clinical concern is choice of medication because therapeutic effectiveness and severe adverse events during treatment vary from patient to patient. In recent years, pharmagenomics has become a consolidated discipline. It informs physicians about molecular subtypes of diseases and which drug is more likely to effectively manage the disease

$40 \quad[3,4]$. Single nucleotide polymorphisms (SNPs) have been proposed as markers to identify the loci associated with complex diseases and their therapeutic treatment [5]. To date, pharmacogenetic research into ADHD has focused mainly on SNPs related to stimulant drugs $[1,2,6]$. In particular, identification in advance of patient-dependent effective drugs (nature and doses) has been correlated with reduced costs and better quality of life.

45 Unfortunately, the incorporation of genomic findings into health care systems is limited as the cost-effectiveness of analytical methods is the main drawback. In fact, the molecular diagnostics field is frequently limited to laborious costly methods that require significant infrastructure and skills, only available for specialized laboratory facilities [7]. The novel generation of diagnostic tools to detect SNPs from a biologic fluid sample is absolutely necessary to develop genuine personalized medicine [8]. The challenge will involve genotyping technologies that integrate conventional properties; i.e. accuracy, robustness, cost-efficiency or automation, with properties such as flexibility, portability or simplicity. Yet relatively few SNPs have been identified as being clinically relevant to treat any given disease. Therefore, the complexity of techniques could be abridged as low-throughput genotyping approaches would be suitable for most clinical scenarios.

Alternative methods to extend SNP genotyping technologies, such as Illumina and Affimetrix platforms, have been proposed and excellently reviewed [9-12]. Certain solutions lie in laboratory approaches, designed for the discrimination of only one SNP of a single patient, or which require equipment that cannot be deployed in resource-limited laboratories.

60 Consequently, some genotyping platforms are far from being actually integrated into routine clinical practice [13].

An advance in SNP genotyping has been made and applied as a pharmacogenetic tool in ADHD treatment in decentralized medical centers. The system is based on microarray assays, performed on polycarbonate chips, and on the later colorimetric detection by a biorecognition process. The use of polymeric substrates is a good solution in research [14], but is key for commercial purposes. Polycarbonate is an excellent material given its properties: mass fabrication under high quality standards, adaptable fabrication of devices, exceptional optical 
properties, wide chemical reactivity for probe anchoring $[15,16]$. By including similar steps to microarray assays performed on glass slides, our methodology uses a fast approach and an inexpensive non fluorescent scanner. Therefore, this method would be accessible for primary or secondary health care scenarios, and is affordable for limited health budgets.

In this study, the discrimination of polymorphisms was achieved by two low-throughput strategies. First, revised allele-specific oligonucleotide hybridization (ASO) was applied using specific probes that were immobilized on polycarbonate slides. Second, allele-specific amplification (ASA) was combined with a hybridization assay as a way to simultaneously detect amplified products.

For its use as a pharmacogenetic tool, three polymorphisms were studied. Relevant clinical evidence has been associated with ADHD drugs, such as methylphenidates and amphetamines, with two SNPs: rs1800544 in the alpha-2 adrenergic receptors (ADRA2A) gene and rs5569 located in the solute carrier family 6 member 2 (SL6CA2) gene [6,17,18]. Information about determining the risk of developing comorbidities associated with ADHD is also in demand [19]. Thus an increased risk for substance abuse has been associated with the genetic variants of polymorphism rs1799971, located in exon 1 of the mu-opioid receptor (OPRMI) gene. This method is examined for the resourceful integration of genetic testing into routine clinical practice and doctor office for ADHD management.

\section{MATERIAL AND METHODS}

Patients. Subjects ( $\mathrm{n}=30$ ) with ADHD symptoms and volunteers were diagnosed by specialist psychiatrists and recruited for the present study according to ethics and with informed consents.

90 Buccal swab and blood samples were collected. The reference genotyping method was the GoldenGate assay with VeraCode Technology (Illumina), which is a high-throughput platform that combines primer extension and ligation to generate allele-specific products, followed by PCR for the amplification and hybridization to codified beads for individual readout.

DNA extraction. Extraction was performed using the PureLink Genomic DNA Mini Kit 95 (Invitrogen), according to the manufacturer's instructions. Briefly, in order to prepare lysate from blood samples, $200 \mu \mathrm{L}$ of fresh blood, $20 \mu \mathrm{L}$ of proteinase $\mathrm{K}, 20 \mu \mathrm{L}$ of RNase and $200 \mu \mathrm{L}$ of kit buffer were added to a microcentrifuge tube. To prepare the human buccal swab lysate, brushes were placed inside the tube with $500 \mu \mathrm{L}$ of PBS, $20 \mu \mathrm{L}$ of proteinase $\mathrm{K}, 20 \mu \mathrm{L}$ of RNase and $500 \mu \mathrm{L}$ of kit buffer. After incubation at $55{ }^{\circ} \mathrm{C}$ for $15 \mathrm{~min}, 500 \mu \mathrm{L}$ of $96-100 \%$ ethanol were added to the tube and the lysate was loaded into the column and centrifuged at $10,000 \mathrm{rpm}$ for 1 minute at room temperature. The flow-through was discarded and the column was washed twice using $500 \mu \mathrm{L}$ of wash buffer 1 and 2 , respectively. Finally, DNA was eluted with $50 \mu \mathrm{L}$ of Tris- $\mathrm{HCl}, \mathrm{pH}$ 8.6. 
Microarraying. Each mixture of streptavidin $(10 \mathrm{mg} / \mathrm{L})$ and biotinylated-surface probe $(50 \mathrm{nM})$ in printing buffer $(50 \mathrm{mM}$ carbonate buffer, $\mathrm{pH} 9.6$ and $1 \%$ glycerol $(\mathrm{v} / \mathrm{v}))$ was transferred to the polycarbonate slide $(25 \mathrm{~mm} \times 75 \mathrm{~mm}$ ). To that end, a non contact printer (AD 1500 BioDot Inc., CA, USA) was used. The drop volume was $50 \mathrm{~nL}$, and working temperature and relative humidity were controlled at $25{ }^{\circ} \mathrm{C}$ and $90 \%$, respectively. The microarray layout consisted of five arrays of $6 \times 6$ dots (ASO approach), or six arrays of $4 \times 6$ dots (ASA approach). Each target gene, positive control and negative control (immobilization and hybridization) had four replicates with a 1-mm track pitch. The design restrictions and oligonucleotides used are described in the Supplementary Material.

115 ASO approach. A triplex PCR reaction was carried out in a total volume of 12.5 1. Each reaction mixture contained 1x DNA polymerase buffer with $2 \mathrm{mM}$ of $\mathrm{MgCl}_{2}$ (Biotools), $200 \mathrm{M}$ of dNTPs (Thermo Scientific), 20 M of digoxigenin-11-dUTP (digoxigenin-X-5-aminoallyl2'-deoxy-uridine-5'triphosphate, Jena Bioscience), $0.4 \mathrm{M}$ of each specific primer, 0.5 units of Taq DNA polymerase (Biotools), and $12.5 \mathrm{ng}$ of the genomic DNA. Cycling conditions were: initial denaturation at $95{ }^{\circ} \mathrm{C}$ for $5 \mathrm{~min}$ followed by 40 cycles of denaturation at $95{ }^{\circ} \mathrm{C}$ for $30 \mathrm{~s}$, primer annealing at $60{ }^{\circ} \mathrm{C}$ for $30 \mathrm{~s}$, elongation at $72{ }^{\circ} \mathrm{C}$ for $30 \mathrm{~s}$, and a final elongation at $72{ }^{\circ} \mathrm{C}$ for $5 \mathrm{~min}$.

A stock solution of saline sodium citrate buffer (SSC 20×) was prepared ( $\mathrm{NaCl} 3 \mathrm{M}$, sodium citrate $300 \mathrm{mM}, \mathrm{pH}$ 7). Prehybridization was performed by dispensing the prehybridization solution (SSC 1.5×, formamide 30\%, and Denhardt's solution $2.5 \times$ ) over microarrays and incubating slides at $50^{\circ} \mathrm{C}$ for 30 minutes in a conventional oven. For hybridization, $2 \mu \mathrm{L}$ of PCR product were mixed with $38 \mu \mathrm{L}$ of hybridization solution (SSC $1.5 \times$, formamide $30 \%$ and Denhardt's solution $2.5 \times$ ). The solution was denatured by heating at $95{ }^{\circ} \mathrm{C}$ for $5 \mathrm{~min}$ and transferred onto the slide surface.

130 After incubation at $37^{\circ} \mathrm{C}$ for $1 \mathrm{~h}$, slides were gently washed for $1 \mathrm{~min}$ with diminishing dilutions of SSC (SSC 1×, SSC 0.5×, SSC 0.05×) and finally with water. Subsequently, a 1-mL mix of 1:2500 sheep anti-digoxigenin antibody (Abcam), and 1:300 anti-sheep antibody conjugated to horseradish peroxidase (Abcam) in phosphate buffered saline solution with 0,05\% Tween-20 detergent, was dispensed onto the slide and incubated for $30 \mathrm{~min}$ at room temperature in the 135 dark. After washing, $1 \mathrm{ml}$ of 3,3',5,5'-tetramethylbenzidine reagent (ep(HS)TMB-mA, SDT, Germany) was spread onto the slide surface and incubated for $8 \mathrm{~min}$ at room temperature. Then slides were washed with water and read. 
ASA approach. Two triplex PCR reactions $(12.5 \mu \mathrm{L})$ were performed by changing the forward 140 primers for the studied polymorphisms. Hence mixture A contained primers with a lower melting temperature and mixture $\mathrm{B}$ contained those with a higher melting temperature. The reaction mixture contained $1 \times$ DNA polymerase buffer without magnesium, $3 \mathrm{mM}$ of $\mathrm{MgCl}_{2}$, $100 \mathrm{M}$ of dNTPs, $10 \mathrm{M}$ of digoxigenin-11-dUTP, $0.3 \mathrm{M}$ of each specific primer, 0.5 units of DNA polymerase and $4 \mathrm{ng}$ of genomic DNA. Cycling conditions were: initial denaturation at 95

$145{ }^{\circ} \mathrm{C}$ for $5 \mathrm{~min}$ followed by 35 cycles of denaturation at $95{ }^{\circ} \mathrm{C}$ for $30 \mathrm{~s}$, primer annealing at $64{ }^{\circ} \mathrm{C}$ (mixture A) and $66^{\circ} \mathrm{C}$ (mixture B) for $30 \mathrm{~s}$, elongation at $72{ }^{\circ} \mathrm{C}$ for $30 \mathrm{~s}$, and final elongation at $72{ }^{\circ} \mathrm{C}$ for $5 \mathrm{~min}$.

The products from both mixtures were hybridized separately in two microarrays on polycarbonate slides, but contained the same oligonucleotides, called common probes. The microarray assay was performed as described above, but the pre-hybridization step was not required and a less stringent hybridization buffer (SSC 1.5×, formamide25\%, and Denhardt's solution 2.5×) and soft washing protocol (SSC 0.1× solution and water) were used. Subsequently, an immunoreaction developed the hybridization products.

155 Detection. Chips were directly scanned (Epson Perfection 1640SU office scanner), and the variation of the reflection properties was measured given the presence of the biorecognition product. If there was no reaction product, the maximum intensity of the reflected beam was collected (background signal). If target gene-probe recognition and subsequent solid deposit formation occurred, the light would strike the product, which would attenuate beam intensity 160 (spot signal). Then gray-scale images (Tagged Image File Format, color depth 16 bit, scale 065535) were generated and the optical intensity signals of each spot were quantified using inhome software. Image processing (feature gridding, addressing, segmentation and quality assurance) was automatically performed in under $5 \mathrm{~min}$.

165 Discrimination criteria. The analysis was declared valid if the controls provided correct responses, and one or two probes of each SNP provided a signal-to-noise ratio above 5. The genotype decision rule was constructed based on the responses of specific probes for each polymorphism. A discrimination index was calculated from the signal of the wild-type (WT) and mutant (MUT) variants, according to the expression (WT - MUT)/(WT + MUT). 


\section{RESULTS}

\section{Allele-specific oligonucleotide hybridization (ASO approach)}

175 A triplex PCR was run for the simultaneous amplification of all the target polymorphisms (rs1800544, rs5569, and rs1799971) using specifically designed primers. The most important variables were $\mathrm{MgCl}_{2}$ concentration, primer concentrations, number of cycles and annealing temperature. Under the selected conditions (Table 1), the amplification factor was $(2.4 \pm 0.3) \times 10^{9}$, as determined by fluorescence measurements .

180 The critical step was the hybridization of the given products with allele-specific probes wildtype and mutant) on polycarbonate slides. The optimization experiments are provided in the Supporting Material and Table 1 summarizes the best conditions. Figure 1 shows the signal-tonoise ratios (SNR) recorded by the scanner, which demonstrates the selectivity of the hybridization pattern. A detectable signal was obtained for the perfect-matched duplexes and a background response (or approached) was acquired for the non matching probes. Therefore, the simultaneous genotyping of three SNPs related to ADHD was possible.

\section{Allele-specific amplification (ASA approach)}

Targeted gene regions were simultaneously amplified using allele-specific primers. For this purpose, two triplex reactions (A and B) were run to obtain specific products for both variants of each polymorphism (rs1800544, rs5569, and rs1799971). The nature of the DNA polymerase, the use of modified primers, and the annealing temperature during thermocycling were optimized (see the Supplementary Material). Under the selected conditions (Table 1), the amplification of the DNA template from a homozygous patient with the incorrect primer was comparable to the negative controls ( $t$-test: $\mathrm{p}$-value $>0.05$ ). The amplification factor for the correct primer was $(1.3 \pm 0.3) \times 10^{9}$.

The detection of ASA products was achieved with a hybridization assay run on a polycarbonate slide following a similar protocol to the ASO approach. In this case, the PCR products of reactions $\mathrm{A}$ and $\mathrm{B}$ were incubated on two microarrays with a specific immobilized probe per gene (common probe independently of the genetic variant). The hybridization conditions (temperature, time, and buffer composition) were optimized by avoiding cross-reactivity between different genes. Under the selected experimental conditions (Table 1), the comparison of the hybridization patterns for both microarrays allowed SNP genotyping (Figure 1). A heterozygous patient led to effective amplification in both reaction mixtures and, consequently, to positive hybridization in both microarrays (SNR > 3). Amplification occurred in a single reaction mixture for a homozygous patient, with a positive result obtained only in the corresponding microarray. 


\section{Method features for SNP genotyping}

210 Different experiments were performed to establish the capabilities of both methods (ASO and ASA) for the genotyping of the three ADHD-related SNPs. Sensitivity was studied by preparing heterozygous mixtures with decreasing percentages of mutant-type DNA compared to the wild type. Mutant DNA was detected up to $4 \%$ (ASO) and 2\% (ASA), which indicates that the system was capable of discriminating both genotypes selectively. Equimolar mixtures of

215 genomic DNA from the WT and MUT homozygous patients were prepared and analyzed. For both approaches, microarray intensities were comparable to the heterozygous genotypes for the three polymorphisms (test $\mathrm{t}: \mathrm{p}>0.1$ ). Intra-day reproducibility and inter-day reproducibility, expressed as the relative standard deviation of spot intensities for triplicate assays, were lower than $15 \%$ and $22 \%$, respectively.

220 The analytical performances, shown in Table 2, demonstrated that both developed methods were adequate as SNP genotyping tools. In fact, the ASO results showed a notably shorter analysis time and lower cost compared to other published or commercially available allele-specific hybridization-based methods $[6,9,20]$. These platforms need long protocols, e.g. overnight hybridization incubations, and also expensive detection equipment. The method developed herein to detect ASA products is much simpler and has a greater multiplexing capability than previously reported methods $[8,21,22]$. In these studies, allele-specific products were detected by electrophoresis or fluorescent dying (real-time or post-amplification). This is also a simpler approach than previous methods that use hybridization assays on glass chips as it avoids chemical activation of the surface, surface blocking and using a fluorescent detector [12].

230 After comparing both the methods developed herein, we found that most assay performances were similar. However, the number of simultaneously analyzed samples was larger in the ASObased method, while the amount of DNA template required was smaller or the assay time was shorter in the ASA-based method. The most marked difference was the efforts made to set-up the multiplex-assay, particularly detection in a microarray format. The design of

235 oligonucleotides and hybridization buffer composition were more critical in the ASO approach. The intrinsic characteristics of ASA genotyping involved a less difficult process to select optimal conditions. Hence the capabilities of the ASA-based method seems less limited than the ASO-based method to extend this genotyping tool to other pharmacogenomic applications.

\section{Analysis of ADHD patients}

The discrimination index was calculated for each studied SNP using the signal-to-noise responses obtained from the three variants (both homozygous and heterozygous individuals). Although different human samples brought about slight variations in the concentration of the 
PCR products, and consequently scored different optical intensities, the discrimination index remained nearly constant for intra-groups (ANOVA test: $p$-value $>0.05$ ). A clear difference was found among the three groups, which allowed the unequivocal genotype assignment of the investigated SNP locus. The heterozygous genotypes produced an intermediate discrimination factor (between -0.3 and +0.3 ), whereas the homozygous ones led to discrimination factors above 0.3 (wild-type) and under -0.3 (mutant), respectively.

250 The method was applied to identify the three loci related to ADHD drugs (rs1800544, rs5569, and rs1799971) in the blinded buccal swab and blood samples. Figure 2 illustrates an example of the microarray images for two patients with a known genotype. As we can see, positive and negative controls provided detectable and background responses, respectively. In all cases, positive optical signals were observed according to the specific polymorphism in the corresponding probes. Nevertheless, the reported genotyping was based on the discrimination factors calculated for each studied SNP, and three populations were perfectly distinguished (Figure 3).

The accuracy of the genotype calls by the microarray-based methods was verified by analyzing the same genomic DNA extracts from ADHD patients using the Illumina Goldengate platform

260 (Supplementary Material). Only five polymorphisms were genotyped erroneously by ASO. The coincidence percentages fell between $90 \%$ (rs5569) and $100 \%$ (rs1800544) for the ASO approach, and were $100 \%$ for the ASA approach. The obtained values were comparable to other low-throughput simple methods that used a single primer/probe per loci $[23,24]$. The reliability of the ASO approach can be enhanced by increasing the number of probes by SNP or including stem probes [6-9]. The comparison to other performances revealed the potential of the technique presented herein. Like other high-throughput platforms, the Goldengate bead-array technology was able to analyze large numbers of SNPs per patient. However, the time required to perform the entire automated process takes about 3 days and involves extremely expensive equipment. In contrast, the developed methods provided genotyping information tailored for the specific disease, e.g. ADHD, in $3.5 \mathrm{~h}$ with equipment that is accessible to, and can be afforded by, almost any laboratory (thermocycler, heating block, and an office scanner or optical microscope).

\section{CONCLUSIONS}

275 The pharmacogenetics applied to personalized medicine aims to identify patterns of genetic variations with cost-effective tools. These systems would make diagnoses in decentralized laboratories, which would streamline management and patient care. This paper addresses this aim developing two fast simple and cheap genotyping methods. Both are based on a simple array assay performed on a planar polycarbonate support for so-called individualized therapy in 
280 ADHD. Although the ASO approach gave adequate results, the ASA approach, based on hybridization with a common probe immobilized on a solid support, yielded better results. The experiments confirmed that the SNP genotyping of ADHD patients, supported by analyzing their genetic code from biological fluids, is actually possible. Nevertheless the present study must be considered the initial step to develop integrated cheap devices, such as lab-on-a-chips, fabricated in plastics and combined with a low-cost detection system.

\section{Acknowledgements}

This research has been funded through projects FEDER MINECO INNPACTO IPT-2011-1132010000, CTQ/2013/45875R and PrometeoII/2014/040 (GVA).

\section{COMPLIANCE WITH ETHICAL STANDARDS}

The authors declare that they have no conflict of interest. Informed consent was obtained from all individual participants included in the study. 


\section{REFERENCES}

1. Cortese S (2012) Eur J Paediatr Neurol 16:422-433

2. Contini V, Rovaris DL, Victor MM, Grevet EH, Rohde LA, Bau CH (2013) Eur Neuropsychopharmacol 23:555-560

3. Gardiner SJ, Begg EJ (2006) Pharmacol Rev 58(3): 521-590

4. Abul-Husn NS, Obeng AO, Sanderson SC, Gottesman O, Scott SA (2014) Pharmacogenomics Pers Med 7:227

5. Altman RB, Flockhart D, Goldstein DB (Eds.) (2012) Principles of pharmacogenetics and pharmacogenomics. Cambridge University Press.

6. Hawi Z, Cummins TDR, Tong J, Johnson B, Lau R, Samarrai W, Bellgrove MA (2015). Mol Psychiatry. 20:289-297

3057 Limaye N (2013). Appl Transl Genomics 2:17-21

8. Manolio TA, Chisholm RL, Ozenberger B, Roden DM, Williams MS, Wilson R, et al. (2013) Genet Med 15:258-267

9. KimS, Misra A (2007) Annu Rev Biomed Eng 9:289-320

10. Lucarelli F, Tombelli S, Minunni M, Marrazza G, Mascini M (2008) Anal Chim Acta 609:139-159

11. Knez K, Spasic D, Janssen KP, Lammertyn J (2014) Analyst 139: 353-370

12. Choi JY, Kim YT, Byun JY, Ahn J, Chung S, Gweon DG, et al.(2012) Lab Chip 12:51465154

13. Ragoussis J (2009) Annu Rev Genomics Hum Genet 10:117-33

315 14. Sethi D, Gandhi RP, Kuma P, Gupta KC (2009) Biotech J 4:1513-1529

15. Bañuls MJ, García-Piñón F, Puchades R, Maquieira A (2008) Bioconjugate Chem. 19: $665-672$

16. Tortajada-Genaro LA, Rodrigo A, Hevia E, Mena S, Niñoles R, Maquieira A (2015) Anal Bioanal Chem 407:7285-7294

320 17. Kieling C, Genro JP, Hutz MH, Rohde LA (2010) Pharmacogenomics 11:407-419

18. Kim, B. N., Kim, J. W., Cummins, T. D., Bellgrove, M. A., Hawi, Z., Hong, S. B., et al. (2013) J Clin Psychopharmacol 33:356-362

19. Carpentier, P. J., Arias Vasquez, A., Hoogman, M., Onnink, M., Kan, C. C., Kooij, J. J. S., et al. (2013) Eur Neuropsychopharmacol 23:448-457

325 20. Zhang Y, Haraksingh R, Grubert F, Abyzov A, Gerstein M, Weissman S, Urban AE (2013) Child Dev 84:34-48 
21. Asari M, Watanabe S, Matsubara K, Shiono H, Shimizu, K (2009) Anal Biochem 386:8590

22. Choi JY, Kim YT, Ahn J, Kim KS, Gweon DG, Seo TS (2012) Biosens Bioelectron, $35: 327-334$

23. Konstantou JK, Ioannou PC, Christopoulos TK (2009) Eur J Hum Genet 17:105-111

24. Sebastian T, Cooney CG, Parker J, Qu P, Perov A, Golova J B., et al. (2014) Clin Chim Acta, 429:198-205 


\section{List of Figures}

Figure 1. Comparison of microarray-based ASO and ASA approaches. Signal-to-noise ratios

340 (SNR) recorded in each probe for a patient with genotypes: GC for rs1800544, GG for rs5569, and AG for rs1799971. WT: wild-type allele-specific probe. MUT: Mutant allele-specific probe. Parentheses indicates the allelic nucleotide.

Figure 2. Microarray images obtained for two patients. (a) ASO probes: $1=\mathrm{NC}, 2=\mathrm{DPC}$, 345 3=HPC, 4=rs5569 (WT), 5=rs1800544 (WT), 6=rs1799971 (WT), 7=rs5569 (MUT), 8=rs1800544 (MUT), 9=rs1799971 (MUT). (b) ASA probes: 1=NC, 2=DPC, 3=HPC, 4=rs5569, 5=rs1800544, 6=rs1799971. Patient 1 genotypes: AG for rs5569, GC for rs1800544, and GG for rs1799971; Patient 2 genotypes: AG for rs5569, GG for rs1800544, and AA for rs 1799971. Abbreviations: NC= Negative control; DPC: Development positive control; HPC:

350 Hybridization positive control; WT: Wild-type allele-specific probe; MUT: Mutant allelespecific probe.

Figure 3. Discrimination of population groups based on ASA results. Boxplot of discrimination index for wild-type homozygous (left), heterozygous (central) and mutant homozygous (right) patients: (a) rs1800544, (b) rs5569, and (c) rs1799971. 


\section{List of Tables}

360 Table 1. Optimal experimental conditions for SNP genotyping by both microarray-based methods.

Table 2. Analytical performances of both microarray-based methods. 